\title{
Physikalische Therapie: Therapiemittel Wasser (2)
}

\author{
Jean-Michel Jeannin
}

Basel, Schweiz

\section{Einleitung}

Die Anwendungsmöglichkeiten des Therapiemittels Wasser sind sehr zahlreich. Ebenfalls zahlreich sind die Indikationen. Der folgende Beitrag legt seinen Schwerpunkt auf die äusserliche Anwendung reinen Wassers - sei es im Rahmen einer schulmedizinischen Behandlung, sei es im Rahmen einer komplementärmedizinischen Anwendung nach Kneipp (Abb. 1). Überschneidungen mit dem bereits besprochenen Thema Balneotherapie (siehe [1]) sind aus Gründen der Systematik unvermeidlich.

\section{Pathophysiologie ausgewählter Krankheiten, die hydrotherapeutisch behandelt werden}

Als Arthrose (engl. osteoarthritis) werden hauptsächlich degenerative Veränderungen eines Gelenks bezeichnet. Neben der altersbedingten Abnutzung kommen als Ursache Unfälle, mechanische Schädigungen und Überlastung infrage. Als Arthritis werden entzündliche Veränderungen eines Gelenks bezeichnet. Die häufigsten Ursachen einer Entzündung sind Unfälle, Übergreifen einer Infektion, Autoimmunkrankheiten und Stoffwechselstörungen, z.B. Gicht. Die Folgen beider Gelenkerkrankungen sind grösstenteils identisch: Einschränkung der Beweglichkeit, Fehlbelastungen der betroffenen Gelenke und starke, chronische Schmerzen.

Bei der Myogelose handelt es sich um eine druckschmerzhafte Verhärtung einzelner Muskelfasern, die sich auch unter Narkose nicht löst. Sie entsteht als Folge von kolloidchemischen Veränderungen oder Spannungszunahme nach längerer Fehlbeanspruchung.

Als Fibromyalgiesyndrom (FMS) wird ein chronischer Schmerzzustand der Weichteile bezeichnet, dessen Ursachen bisher ungeklärt sind. Gegenwärtig wird daran gearbeitet, das bisherige diagnostische Kriterium der

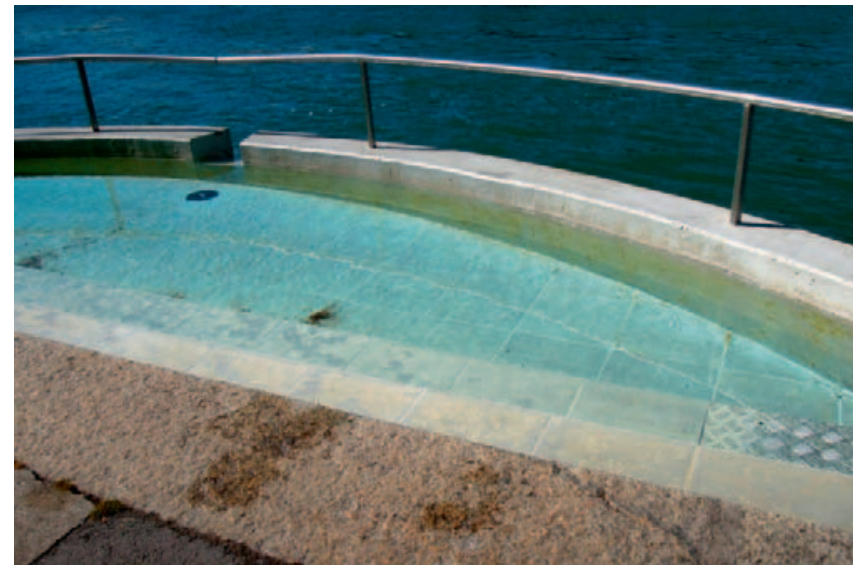

Abb. 1. Kaltwasserbecken am Rhein für Wassertreten nach Kneipp.

druckschmerzempfindlichen Punkte (Tender Points) zu verlassen. Das FMS ist häufig mit einer Depression vergesellschaftet.

\section{Wirkungsweise der Hydrotherapie}

Die Hydrotherapie entfaltet ihre Wirkung über eine Palette von Einzelfaktoren, wozu die Temperatur des Wassers, der hydrostatische Druck beim Eintauchen, der Auftrieb (beim Eintauchen) sowie der Reibungswiderstand bei Bewegung im Wasser gehören (Abb. 2). Im Fall einer Wasserstrahlmassage kommt der Druck des Wasserstrahls als weiteres Element dazu. Die Effekte einer Hydrotherapie beruhen auf zwei Wirkqualitäten: der Temperatur des angewendeten Wassers (thermische Reize) und der Entlastung der behandelten Körperregion durch den Auftrieb. Die thermischen Reize wirken bei wiederholter (serieller) Anwendung; sie lösen funktionell-adaptive Umstellungen aus. Der Auftrieb wird unmittelbar für Bewegungsübungen genutzt [2]. Srámek et al. [3] fanden in ihrer Studie an gesunden, freiwillig teil-

\section{KARGER}

Fax +497614520714

Information@Karger.com

www.karger.com

\section{(c) 2015 S. Karger GmbH, Freiburg}

$1015-0684 / 15 / 0274-0215 \$ 39.50 / 0$

www.karger.com/szg 


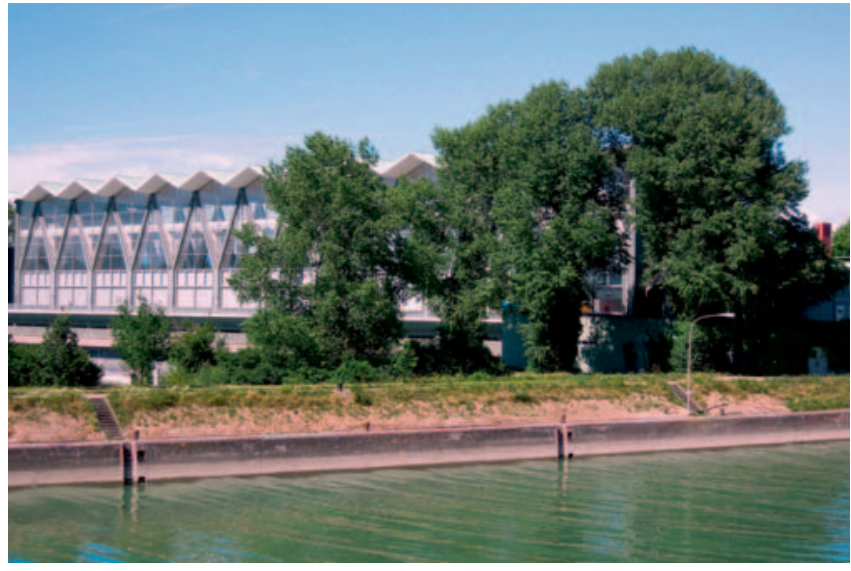

Abb. 2. Kraft des Wassers: Maschinenhaus des Kraftwerks Birsfelden.

nehmenden jungen Männern die Hypothese bestätigt, dass die Veränderung physiologischer Parameter wie Blutdruck und Herzfrequenz beim Eintauchen in Wasser hormonell gesteuert werden, im Unterschied zum Einfluss der Kälte, die den Sympathikotonus erhöht [3].

\section{Klinische Studien}

Assis et al. [4] verglichen in einer randomisierten und kontrollierten klinischen Studie an 60 FibromyalgiePatienten im Alter von 18 bis 60 Jahren die Wirksamkeit eines Trainings im Wasser mit derjenigen eines Trainings an Land. Die Patienten führten die Übungen jeweils an der anaeroben Schwelle aus. Beide Methoden erwiesen sich als gleich wirksam bezüglich der Schmerzreduktion und der Verbesserung der Stimmung. Der Trainingseffekt war für beide Gruppen ähnlich gross. Die Wirksamkeit auf die emotionellen Aspekte wurde von den Autoren im Fall der Übungen im Wasser jedoch als überlegen bewertet [4]. In einer klinischen Studie an 61 Patienten waren die messbaren Effekte der von den Patienten zu Hause durchgeführten aeroben Übungen gleich gut wie die von unter Anleitung eines Physiotherapeuten durchgeführten hydrotherapeutischen Übungen (Verminderung der Schmerzen, Verbesserung der Stimmung, Verbesserung der Funktion). Die Effekte dauerten jedoch in der Gruppe der von einem Physiotherapeuten behandelten Patienten länger an [5]. In einer einfach-blinden klinischen Studie wurde der Verlauf einer Hüftarthrose oder einer Kniearthrose nach einer sechswöchigen hydrotherapeutischen Behandlung versus keine Behandlung untersucht. Die Studienpopulation bestand aus 71 Patienten. Geprüft wurden die Schmerzintensität sowie funktionelle Parameter. Eine Verbesserung der Funktionalität des betroffenen Gelenks wurde bei 75\% der behandelten Patienten beobachtet, eine Abnahme der Schmerzen bei 72\%. In der Kontrollgruppe ergab sich eine Besserung beider Parameter bei $17 \%$ der Patienten aus der Kontrollgruppe. $84 \%$ der Teilnehmer führten die Behandlung am Ende der Studie freiwillig weiter [6]. Bewegungsübungen im Wasser waren bezüglich der Schmerzreduktion und Funktionsverbesserung deutlich besser wirksam als Bewegungsübungen an Land. So lautete das Resultat einer randomisierten klinischen Studie von Silva et al. [7] mit 64 Patienten, die an einer Kniearthrose litten. Keine Überlegenheit der Hydrotherapie gegenüber Übungen an Land fanden Foley et al. [8] in ihrer Studie an 105 Patienten, die an einer Knie- oder Hüftarthrose litten. Die gesamte Verbesserung der Funktionen war in beiden Gruppen ähnlich gross. Es bestanden jedoch Unterschiede bezüglich der einzelnen Parameter [8]. Green et al. [9] behandelten ambulante Patienten mit einer Hüftarthrose mittels verordnetem Training zu Hause und zusätzlicher Hydrotherapie oder ohne Zusatztherapie. Unabhängig von Alter, Geschlecht und Schweregrad der Arthrose bestand kein statistisch signifikanter Unterschied zwischen den Ergebnissen der beiden Gruppen. Hydrotherapie als zusätzliche Behandlung brachte nur einen geringen zusätzlichen Nutzen [9] . Einen ganz anderen Ansatz verfolgten Schencking et al. [10]: Die Autoren verglichen in einer randomisierten dreiarmigen kontrollierten Pilotstudie mit 30 Patienten mit Arthrose des Hüft- oder des Kniegelenks die Wirksamkeit einer Hydrotherapie nach Kneipp mit der einer konventionellen Physiotherapie. Gruppe 1 wurde mit Hydrotherapie nach Kneipp behandelt, Gruppe 2 mit konventioneller Physiotherapie. Gruppe 3 diente als Kontrollgruppe und erhielt beide Behandlungen. Die besten Ergebnisse (Schmerzminderung, Verbesserung der Funktion) zeigte Gruppe 1, gefolgt von Gruppe 3 und Gruppe 2. Nach Ansicht der Autoren bedarf es zur Sicherung der Ergebnisse einer grösseren Stichprobe [10]. Eine klassische Kneipphydrotherapie mit kurz andauernden Kältereizen vermochte die Symptome eines Menopausen-Syndroms bei 60 Patientinnen deutlich zu vermindern. Das Ergebnis muss allerdings mittels weiterer Studien erhärtet werden [11]. Eine leichte Verbesserung der Lungenfunktionsparameter und eine deutliche Senkung der Anzahl an Infektionen von 20 COPD-Patienten (17 männlich, 3 weiblich) wurde in einer Untersuchung der Wirksamkeit von seriellen Obergüssen nach Kneipp im Rahmen einer Dissertation beobachtet. Die Wirkung auf die Infektionsanfälligkeit («Th1-Reaktion») entspricht der umgangssprachlichen Abhärtung [12].

\section{Ergänzende Bemerkungen zur Kneipp-Kur}

Eine klassische Kneipp-Kur umfasst neben der Anwendung von Wasser die Elemente Bewegung, Ernährung, Phytotherapie und Innere Ordnung. Es werden somit auch Fragen der Lebensführung angegangen. Der be- 


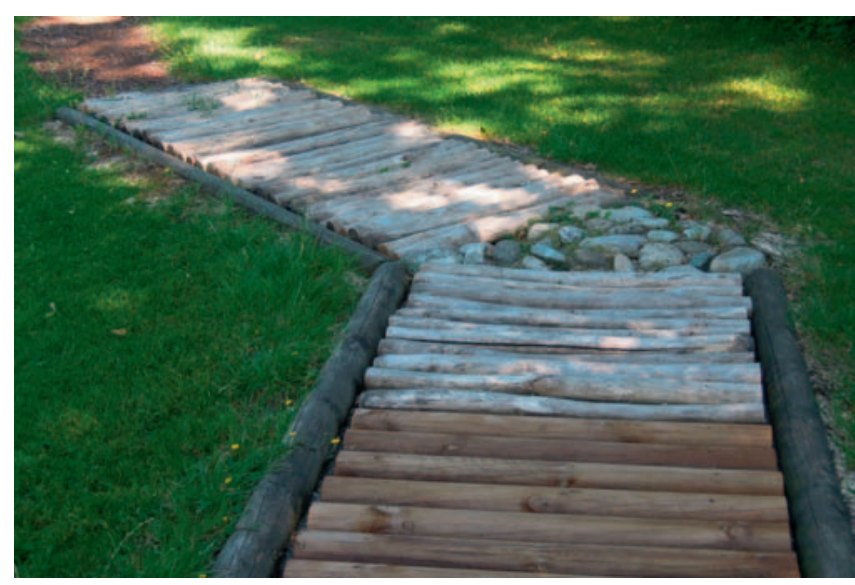

Abb. 3. Barfusspfad nach Kneipp, Birsfelden.

rühmte Barfusspfad (Abb. 3) kann am besten dem Element «Bewegung» zugeordnet werden. Der Autor konnte in einem Selbstversuch die hohe Empfindlichkeit der Fusssohlen erleben. Nach der Rückkehr in die Schuhe blieben längere Zeit ein sehr angenehmes Gefühl und eine erhöhte Wahrnehmung des Bodens erhalten. Eine sehr genaue Wahrnehmung des Bodens ist für das Gehen essenziell: «Motorik und Sensorik bedingen sich gegenseitig und sind als Einheit zu sehen ...» [13].

\section{Schlussbemerkung}

Die Hydrotherapie bewegt sich an der Grenze zwischen Schulmedizin und Komplementärmedizin. Die zitierte und konsultierte Literatur zeigt die Wirksamkeit der Hydrotherapie als schulmedizinische Behandlung sowie der Hydrotherapie nach Kneipp als komplementärmedizinische Behandlung. Für die Patienten erwiesen sich Behandlungsmethoden, die sie selbst anwenden können, und solche, die weitere therapeutische Massnahmen umfassen, als attraktiv. Das subjektive Befinden wird in der Tendenz bei komplementärmedizinischen Behandlungen als besser beurteilt, was plausibel scheint.

\section{Literatur}

1 Jeannin J-M: Physikalische Therapie: Therapiemittel Wasser (1). Schweiz Z Ganzheitsmed 2015;27:92-94.

2 Glaesener JJ: 3.9 Hydrotherapie; in Gutenbrunner C, Glaesener JJ: Rehabilitation, Physikalische Medizin und Heilverfahren. Heidelberg, Springer, 2007, pp 80.

3 Srámek $\mathrm{P}$, et al: Human physiological responses to immersion into water of different temperatures. Eur J Appl Physiol 2000;81: 436-442.

4 Assis MR, et al: A randomized controlled trial of deep water running: clinical effectiveness of aquatic exercise to treat fibromyalgia. Arthritis Rheum 2006;55:57-65.

5 Evcik D, et al: Effectiveness of aquatic therapy in the treatment of fibromyalgia syndrome: a randomized controlled open study. Rheumatol Int 2008;28:885-890.
6 Hinman RS, et al: Aquatic physical therapy for hip and knee osteoarthritis: results of a single-blind randomized controlled trial. Phys Ther 2007;87:32-43.

7 Silva LE, et al: Hydrotherapy versus conventional land-based exercise for the management of patients with osteoarthritis of the knee: a randomized clinical trial. Phys Ther 2008;88:12-21.

8 Foley A, et al: Does hydrotherapy improve strength and physical function in patients with osteoarthritis - a randomised controlled trial comparing a gym based and a hydrotherapy based strengthening programme. Ann Rheum Dis 2003;62:1162-1167.

9 Green J, et al: Home exercises are as effective as outpatient hydrotherapy for osteoarthritis of the hip. Br J Rheumatol 1993;32:812-815.
10 Schencking M, et al: A comparison of Kneipp hydrotherapy with conventional physiotherapy in the treatment of osteoarthritis: a pilot trial. J Integr Med 2013;11:17-25.

11 Ortiz M, et al: Kneipp hydrotherapy for menopausal symptoms - results of a pilot study. FACT 2006;11(suppl 1):33-34.

$>12$ Goedsche K: Serielle Kaltwasserreize (Kneipp'scher Oberguss) bei Patienten mit chronisch obstruktiver Bronchitis (COPD). Forsch Komplementärmed 2007;14:158-166.

13 Sensomotorik; in Haus K-M: Neurophysiologische Behandlung bei Erwachsenen. Grundlagen der Neurologie, Behandlungskonzepte, alltagsorientierte Therapieansätze. Berlin, Springer, 2005. 